\title{
De la dispersión a la centralización de las políticas públicas en el sector rural de México
}

\author{
From dispersion to centralization of public policies \\ in the rural sector of México
}

Julio Baca del Moral ${ }^{1}$, Venancio Cuevas-Reyes ${ }^{2}$ y Pilar Barradas-Miranda ${ }^{3}$

Fecha de recepción: 5 de mayo de 2020

Fecha de aceptación: 24 de septiembre de 2020

1- Nacionalidad: Mexicana. Grado: Doctor en Estudios Rurales, orientación Desarrollo Rural. Adscripción: Universidad Autónoma Chapingo. DORCID: http://orcid.org/0000-0002-2197-2517. Correo electrónico: julio.baca56@gmail.com

2- Nacionalidad: Mexicana. Grado: Doctor en Problemas Económico-agroindustriales. Adscripción: Universidad Autónoma Chapingo /Instituto Nacional de Investigaciones Forestales, Agrícolas y Pecuarias (INIFAP-México). (iDORCID: http:// orcid.org/0000-0001-9946-3942. Correo electrónico: cuevas.venancio@gmail.com

3- Nacionalidad: Mexicana. Grado: Doctora en Estudios Organizacionales. Adscripción: Universidad de Quintana Roo. (D) ORCID: http://orcid.org/0000-0002-4210-4874. Correo electrónico: pilarbarradas@gmail.com 


\section{Resumen}

El objetivo del trabajo es analizar la implementación de dos de los programas estelares de la administración federal actual para el sector rural, con el enfoque del ciclo de las políticas públicas. De 2017 a 2019 se realizó un estudio en 24 comunidades de seis estados del país, los resultados reflejaron la dispersión de recursos en más de 100 programas; en las comunidades estudiadas, los beneficiarios solo recibian dos o tres de ellos. En el nuevo sexenio se ha percibido la falta de información precisa sobre el acceso a los programas, en la opinión pública no se percibe un diseño y planeación de políticas que impacten de manera concreta en el fomento productivo; se continúa y profundiza en políticas de transferencias y asistencia social. Se concluye que continúa un esquema similar al anterior gobierno en la definición, diseño e implementación de políticas públicas para el campo.

Palabras clave: desarrollo rural, políticas públicas, planeación participativa.

\section{Abstract}

The objective focuses on analyzing the implementation of two public policies for the rural sector in the new federal public administration, under the focus of the public policy cycle. From 2017 to 2019 a study was carried out in 24 communities in six states of the country, the results reflected the dispersion of resources in more than 100 programs; in the communities studied, the beneficiaries only received two or three of them. In the new six-year period, the lack of precise information on access to programs has been perceived. Public opinion does not perceive a design and planning of policies that specifically impact productive promotion; it continues and deepens in transfer policies and social assistance. It's concluded that a similar scheme to the previous government continues in the definition, design and implementation of public policies for the field.

Keywords: rural development, public policies, participatory planning. 


\section{Introducción}

$\mathrm{D}$

urante las últimas tres décadas, el gobierno ha puesto mayor énfasis en el apoyo a la agricultura de exportación y menos a la pequeña agricultura familiar. Los precios subsidiados de alimentos importados y la ineficacia en la aplicación de políticas públicas que pretenden atender a las pequeñas unidades de producción familiar, sin considerar la enorme heterogeneidad de su conformación, sus prácticas productivas y su entorno natural y socio cultural; han dejado en la pobreza a más de tres millones de productores. A esto se suman las políticas económicas de corte neoliberal y los programas agroalimentarios burocratizados e inefectivos, presupuestos insuficientes y mal aplicados, bajas inversiones en bienes públicos, debilidad de las instituciones gubernamentales, con multiplicidad de acciones duplicadas y escasa o nula coordinación entre dependencias e instituciones, además, de un creciente nivel de corrupción. "Estos factores han inhibido el desarrollo del sector, aumentado la pobreza rural, afectando al 20\% de la población del país y dejando sin oportunidades a los jóvenes rurales, exponiéndolos a actividades ilícitas y fomentando su emigración" (Villalobos, 2018, p. 129).

A nivel nacional, los índices que miden la pobreza alimentaria representan una problemática difícil de solucionar. De acuerdo con datos del Consejo Nacional de Evaluación de Política de Desarrollo Social, CONEVAL (2016) del total de la población mexicana $43.6 \%$ se encuentra en estado de pobreza, es decir 53.4 millones de personas, de las cuales 11.4 millones se encuentran en pobreza extrema. En cuanto a la dimensión alimentaria de la pobreza, CONEVAL (en el mismo estudio) reportó que $20.1 \%$ del total de la población, 24.6 millones de personas, se encuentran en carencia por acceso a la alimentación, aun cuando los recursos económicos para el sector rural son amplios. En 2016, en el Programa Especial Concurrente (PEC) los recursos aprobados ascendieron a $352 \mathrm{mil} 842 \mathrm{mdp}$, para el 2018 la cantidad de recursos al campo fue superior a 330 mil mdp, en más de 150 programas desglosados en el PEC. Es decir, la pobreza persiste aún y cuando existió una gran cantidad de gasto para el sector rural, disperso en más de 150 programas.

Esta nueva administración 2018-2024 está realizando lo que denomina una Cuarta Transformación (4T) del país; para el campo ha integrado una buena cantidad de dichos programas (PEC, 2019); también, concentra la toma de decisiones. En este trabajo se hace un análisis documental de dos programas de la 4T: Producción para el Bienestar (Secretaría de Agricultura y Desarrollo Rural, SADER) y Sembrando Vida (Secretaría del Bienestar, SB). Con ello se observan varios aspectos novedosos y loables, y también viejos problemas en las políticas públicas (PP).

El planteamiento central de este análisis es llamar la atención del cambio respecto de una gran dispersión de programas para el campo (más de 150 en 2018) a una concentración de los mismos y la centralización en la toma de decisiones a través de los llamados "super delegados" y Presidencia de la República. Para ello, se abordan los casos de "Producción para el bienestar" de la SADER y el de "Sembrando vida" de la Secretaria del Bienestar (SB). Partimos de la propuesta general que debiera seguirse en el diseño y ejecución de las políticas públicas, así como de los resultados y las recomendaciones realizadas en un estudio que desarrollamos entre 2017 y 2019. Como se mencionó antes, el objetivo consiste en analizar la implementación de dos políticas públicas para el sector rural en el nuevo sexenio, comparándolo con lo que sucedía en años previos y con ello identificar si la nueva definición de políticas sigue las etapas 
del ciclo de las políticas públicas. La hipótesis de trabajo se refiere a que, si bien con la nueva administración los programas de atención al sector rural en México se encuentran concentrados, los problemas de operación, concreción y obtención de resultados siguen el mismo esquema anterior y no se logran los resultados esperados a nivel de unidad de producción rural.

La investigación empírica se realizó en seis estados del país que cuentan con pequeña agricultura familiar. La información se obtuvo como parte del proyecto "PESA y CNCH las sinergias para potenciarlas”, financiado por CONACYT 2015-01-732. Durante el año 2017, se aplicó una encuesta para caracterizar la agricultura multifuncional familiar, en más de 400 unidades de producción de seis entidades del país: Chiapas, Oaxaca, Quintana Roo, San Luis Potosí, Veracruz y Yucatán.

La estructura del trabajo se presenta de la siguiente forma: en el primer apartado se describe el marco conceptual utilizado, posteriormente se rescatan los aprendizajes y propuestas del estudio sobre agricultura multifuncional y políticas públicas del proyecto mencionado, siguiendo el enfoque propuesto por Baca y Cuevas (2018) de lograr una mayor vinculación de los programas con pequeños productores rurales. En un segundo apartado se analizan los programas ya mencionados: "Producción para el Bienestar" y "Sembrando Vida", en 2019, después se presenta una sección sobre discusión de los resultados encontrados y, finalmente, algunas reflexiones a manera de conclusiones.

\section{Políticas públicas: concepto, agenda y ciclo}

Para centrarnos en el tema de este trabajo, cabe especificar un marco conceptual: ¿Qué es el Estado? y ¿Qué es el gobierno? baste recordar: el Estado es la estructura de nación que cada país se da a sí mismo, estableciendo las bases en su constitución. En México, está conformado por tres poderes: ejecutivo, legislativo y judicial. Mientras que el gobierno, es la administración que cambia cada seis años, el poder legislativo, representado por la Cámara de Senadores y de Diputados, la cual por su parte, cambia cada tres años. Se clasifican en tres órdenes de gobierno: federal, estatal y municipal.

El gobierno es quien dicta y elabora la agenda de asuntos públicos a atender durante su administración, sin embargo, como señala Aguilar (1993, pp. 21-23) "No todos los problemas logran llamar la atención gubernamental y despertar su iniciativa, asimismo, no todos logran con la misma facilidad y certeza formar parte del temario de los asuntos públicos y colocarse entre los asuntos prioritarios del gobierno". Es decir, no cualquier problema o situación logra establecerse como prioritario en la agenda pública, la instalación de problemas públicos en ésta se constituye con aquellos problemas o situaciones que identifica el gobierno como relevantes y a los cuales desea incluir (sea por presión de grupos o convencimiento), en sus políticas públicas, programas y estrategias, entonces así se constituye la agenda de gobierno. La cual se define "como el conjunto de problemas, demandas, cuestiones, asuntos, que los gobernantes han seleccionado y ordenado como objetos de su acción y, más propiamente, como objetos sobre los que han decidido que deben actuar o han considerado que tienen que actuar" (Aguilar, 1993, p. 30). Una vez que se determina el problema a resolver, este debe ser incorporado a la agenda del gobierno, ya que como señala Peters (2002, p. 472): "es solamente cuando un problema es colocado en la agenda y puesto a disposición para discusión, que las fuerzas del cambio tienen alguna oportunidad de triunfar". En este sentido, "La razón de ser del análisis de políticas es construir una definición del problema, y por 
ende, la política correspondiente que indique a la comunidad política lo que realmente pueden querer y desear porque es lo que razonablemente pueden alcanzar" (Aguilar, 1992a, p. 72). Las PP son aquellas estrategias encaminadas a resolver problemas públicos (Lasswell, 1992). Meny y Thoenig (1992, p. 90) definen a la política pública como "un programa de acción gubernamental en un sector de la sociedad o en un espacio geográfico". Por tanto, desde el punto de vista teórico la definición del problema y las causas que lo originan resulta una de las etapas más relevantes en el ciclo de las políticas públicas, ya que:

...una política pública implica el establecimiento de una o más estrategias orientadas a la resolución de problemas públicos, así como a la obtención de mayores niveles de bienestar social resultantes de procesos decisionales tomados a través de la coparticipación de gobierno y sociedad civil, en donde se establecen medios, agentes y fines de las acciones a seguir para la obtención de los objetivos señalados. (González, 2005, p. 110)

En teoría, el diseño de las políticas públicas debiera seguir una serie de fases y establecer las reglas del juego entre los diferentes sectores participantes (público, privado y social), a partir de las cuales deben establecerse, ubicarse y acordarse las situaciones sociales que han de ser consideradas como problemas públicos a atender, es decir, identificar lo que Oszlak y O'Donnell (1995) llaman "cuestiones socialmente problematizadas". Diversos autores han identificado distintas fases o etapas en el diseño o ciclo de elaboración de las políticas públicas, estas etapas se encuentran vinculadas porque todas ellas se retroalimentan.

En concordancia con varios autores como Aguilar (1992b), Vallés (2000), González (2005), Wastell (2006) y Flores (2013) entre otros, se establece el llamado ciclo de las PP; es decir, que toda política pública ha de contar con al menos cuatro grandes etapas: diagnóstico, diseño, implementación y evaluación.

Es importante iniciar con un diagnóstico que permita conocer concretamente la situación y las variables que inciden en la problemática que se intenta resolver o evitar; es conveniente elaborar el diagnóstico a través de ejercicios participativos con quienes se pretende sean los sujetos de apoyo, para conocer de primera mano los problemas y las posibles soluciones que se perfilen en la experiencia de quienes se encuentran en dicha situación; se suman a esta práctica: expertos en la materia, para conocer otra perspectiva de la problemática y, las instancias a quienes correspondería la gestión y seguimiento del programa. De manera simultánea se revisan experiencias similares tanto a nivel local como nacional o internacional, para establecer parámetros que coadyuven a visualizar de antemano algunos obstáculos que pudieran presentarse.

Luego, para la adecuada planeación se requiere en principio, establecer objetivos y metas de manera precisa y concreta, definir de manera consistente quiénes serán los participantes (sujetos y actores), desde los sujetos de apoyo, hasta los técnicos y servidores públicos en los niveles de decisión; si se realiza un mapa de actores, es posible ubicar de antemano un diagrama de flujo que sea lo más ágil posible y permita una gestión y aplicación eficiente, en tiempo, forma y calidad.

Diseño. En esta fase debe establecerse específicamente la región o regiones donde se ejecutarán las actividades del programa y con ello los esquemas de capacitación que serán indispensables para que todos los participantes comprendan, tanto el alcance como la limitación propia de la política a establecer.

Implementación o ejecución. En consonancia de lo desarrollado, se debe contemplar el logro de las metas planteadas, a través del seguimiento en la ejecución, en los distintos niveles y actores involucrados. Aquí, se opera el seguimiento puntual de lo planeado, se aplican algunos indicadores que se hayan con- 
signado para verificar la ejecución y se documentan los primeros resultados.

Evaluación. A lo largo del proceso se recomienda hacer un seguimiento para tener certeza sobre la trayectoria del programa, la evaluación puede hacerse en distintos momentos, desde el diseño hasta al término de la etapa o etapas consideradas en la planeación; se comparan los resultados con base en el nivel de logro para la resolución de la situación problemática y/o la mejoría parcial lograda con el proceso. Evaluar una política pública es de fundamental importancia, ya que por lo general ocurren desviaciones en la ejecución, y en ocasiones se ignoran los impactos específicos que genera, así como los acuerdos necesarios para conseguir una política pública de calidad, Aguilar (2008), afirmó lo siguiente:

...el análisis de PP es una actividad intelectual multidimensional, de muchas capas y muchos puntos cardinales. El rigor intelectual, metodológicamente cultivado, es una condición necesaria, así como lo es también la capacidad de hacerse nuevas preguntas y la disposición a explorar nuevos caminos. Crucial es la voluntad de no aceptar que la política se vuelva sólo un hecho de poder o de conveniencia, insubordinado a las exigencias de la razón valorativa y empírica e indiferente a las aspiraciones de una vida en sociedad segura, próspera y justa. (p. 22)

Este proceso es continuo así, se pueden realizar varias actividades en forma simultánea, mismas que deben quedar documentadas a fin de contar con evidencias para revisar y poder re-establecer cursos de acción.

Respecto al enfoque de las PP basadas en evidencias (PPBE) cabe mencionar que el término referido a la implementación de políticas basadas en evidencias se desarrolló en los 90 en el sector salud (Johnson y Austin, 2005). El tema medular de este enfoque es el concepto de "evidencias", al respecto Sutcliffe y Court (2006, p. 2) señalan, “... lo que resulta claro a partir de la bibliografía disponible es que evidencia es un término ambiguo. Creemos que la política basada en la evidencia debe estar basada en la evidencia sistemática, es decir, evidencia basada en investigaciones". Por otra parte, una aproximación transversal a este enfoque lo propone Buchanan (2009, p. 45), quien argumenta “... la necesidad de que los programas sociales, tratamientos, intervenciones, servicios y acciones en general que se apliquen a cualquier contexto, deberían estar avalados por la mejor evidencia científica posible".

De acuerdo con Davis (2004, p. 3) el enfoque de PPBE “ayuda a las personas a tomar decisiones bien informadas sobre políticas, programas y proyectos al poner la mejor evidencia disponible de la investigación en el centro del desarrollo e implementación de políticas". En este sentido, "el objetivo de las PPBE se basa en la premisa de que las decisiones políticas deben estar mejor informadas a partir de la evidencia disponible, y debería incluirse un análisis racional. Esto es porque se considera que las políticas y prácticas basadas en la evidencia sistemática han producido mejores resultados" (Sutcliffe y Court, 2006, p. 2).

Sutcliffe y Court (2006), a partir de un análisis bibliográfico, señalan lineamientos importantes para el enfoque de políticas basadas en evidencias, para estos autores resulta claro que: 1) el uso de la evidencia importa: un mejor uso de la evidencia en las políticas y las prácticas puede ayudar a reducir la pobreza y mejorar el rendimiento económico en los países en desarrollo, 2) las políticas deben estar informadas por una amplia gama de evidencia, no sólo por datos empíricos. Entre los temas claves, se encuentran la calidad, la credibilidad, la relevancia y el costo de la política, 3) la evidencia es necesaria en todos los distintos componentes del proceso de políticas, y de diferentes modos en cada componente, 4) varias limitaciones (tiempo, 
capacidad, costo) afectarán los mecanismos disponibles para movilizar la evidencia para las políticas en los países en desarrollo, y 5) los procesos políticos son inherentemente políticos: aunque algunos países en desarrollo cuentan con contextos complicados, un número aún mayor debe explorar los enfoques de las PBE.

El enfoque de PPBE no está libre de señalamientos en el sentido de que "la evidencia científica debe ser complementada con otro tipo de información como la originada en los procesos de operación y en las esferas de la política (politics). Asimismo, es importante considerar a la evidencia científica, como un incentivo, para debatir cuál puede ser el instrumento de política más idóneo para hacerle frente al problema detectado" (Flores-Crespo, 2013, p. 286). Pese a lo anterior, este es un enfoque que, de acuerdo a las políticas públicas para el campo mexicano y, dado su escaso impacto en el otorgamiento de resultados, puede aplicarse como parte de un soporte técnico, para coadyuvar en cualquiera de las fases del ciclo de políticas públicas mencionadas con anterioridad.

Es así que, el enfoque de las políticas basadas en evidencias, se fundamenta a través del conocimiento de situaciones particulares obtenidas con un nivel alto de análisis y rigor científico. "La evidencia puede sustentar el proceso desde la identificación del problema, la formulación, y la implementación ... asimismo, puede determinar el impacto de la política y los ajustes necesarios para mejorar sus resultados y aplicabilidad en distintas poblaciones (Gutiérrez et al., 2016, p. 580).

\section{Retrospectiva de políticas públicas: mucho gasto y poco efecto}

A continuación, se presenta la información resultado del proyecto de investigación sobre pequeñas unidades familiares de producción rural financiado por el CONACYT (PDCPN 2015.01.732). Se utiliza el enfoque de "diseño de políticas basado en evidencias" propuesto por Davies (2004) y Sutcliffe y Court (2006).

Las categorías que se consideraron para el análisis fueron: conocimiento de los programas, y cobertura efectiva de los mismos, es decir, de los programas existentes, cuántos realmente operan de manera regular, con beneficiarios en las localidades rurales y los resultados logrados en comparación a los esperados. Con la perspectiva de las evidencias encontradas se identificaron los problemas existentes en el diseño, planeación, ejecución y evaluación de las políticas públicas destinadas al sector rural en México. El proyecto se enfocó de forma territorial y se buscó la posibilidad de hacer sinergias con las políticas establecidas por el gobierno federal, sobre todo en aquellos programas que atienden las necesidades locales de los pequeños agricultores (menos de 5 has), y que les permitan plantearse un desarrollo sustentable con equidad y respeto a su cultura. Como se ha señalado, el trabajo se ubicó en seis entidades del país: Chiapas, Oaxaca, Quintana Roo, San Luis Potosí, Yucatán y Veracruz. El tamaño de muestra consideró 402 unidades de producción familiar distribuidas en 24 comunidades en los seis estados antes mencionados.

La información se obtuvo con encuestas directas a unidades de agricultura familiar multifuncional (UAMF) en 2017, las preguntas se orientaron en los ejes: técnico productivo, socio económico, cultural, ambiental, programas públicos, organizaciones y género. Las encuestas se realizaron con la aplicación KoBoCollect, el uso de esta aplicación permite aplicar las encuestas cara a cara con los productores sin contar con servicio de Internet en los dispositivos electrónicos utilizados (celular o tableta), esto es muy importante cuando se aplican encuestas en zonas territorios rurales dispersos y lejanos, como es la gran 
mayoría de nuestro país. La sistematización se efectuó con el programa Qlik que permite el análisis asociativo de los diferentes datos y variables en estudio (Barradas, 2018). Además, tiene la ventaja de que se cuenta con el acceso instantáneo a los datos, es flexible en el uso y puede actualizarse en tiempo real, presenta hojas analíticas y visualizaciones predeterminadas, por lo que se pueden generar gráficos sin requerir capacitación especializada.

En sexenios anteriores existía una amplia oferta de programas en México, 150 relacionados con la agricultura y la alimentación, cuya responsabilidad correspondía a diferentes instancias gubernamentales. No obstante, en las encuestas aplicadas a estas 402 unidades de producción familiar, solamente se registró el apoyo de nueve de ellos, mismos que se enuncian a continuación.

El Programa de Empleo Temporal (PET), PROSPERA, el Programa de Apoyo Alimentario (PAL), y el denominado 65 y más, todos ellos operados por la Secretaría de Desarrollo Social (SEDESOL). Otros cuatro programas a cargo de la Secretaría de Agricultura, Ganadería, Desarrollo Rural, Pesca y Alimentación (SAGARPA): el Programa de Seguridad Alimentaria (PESA), Proagro Productivo (antes PROCAMPO), Programa de Apoyo para Productores de Maíz y Frijol (PIMAF) y Programa de Producción Pecuaria Sustentable y Ordenamiento Ganadero y Apícola (PROGAN). Además, por parte de la Comisión Nacional para el Desarrollo de Pueblos Indígenas (CNDPI) se evidenció solamente uno, el Programa para el Mejoramiento de la Producción y Productividad Indígena (PROins).

De los nueve programas que efectivamente llegaban a las familias rurales, el denominado PROSPERA tenía mayor cobertura; en segundo lugar, el PESA; después el PROAGRO; en cuarto lugar "65 y más" $y$, en quinto lugar, el PAL. El resto de programas: PIMAF, PROGAN, PROins, y PET contaban con una cobertura menor.

Como puede apreciarse, en comparación con la oferta reportada a nivel nacional, los programas que efectivamente atendían a los productores de la agricultura multifuncional diversificada en los seis estados, eran muy pocos, dispersos y más en la vertiente asistencialista. Esto se evidencia en la limitada cobertura de aquellas iniciativas que podrían incrementar la producción y capitalizar a las familias; es decir, las políticas públicas para este tipo de productores se enfocaban en mantenerlos en la misma situación de carencia de oportunidades, y no en orientar hacia la autogestión y direccionamiento productivo. A nivel estatal, en promedio solo dos programas otorgaban apoyo a las UAMF; mínimo recibían apoyo de un programa por unidad y máximo podían recibir apoyo de cuatro programas. En las comunidades analizadas en el estado de Veracruz, por ejemplo, el máximo de apoyos de programas recibidos fue de tres.

Respecto al número de beneficiarios, en la revisión de los programas se identificó que de 792 sujetos de apoyo de los programas públicos (en el entendido de que una unidad de producción puede tener más de un apoyo) cuatro programas de asistencia social eran promovidos por la SEDESOL ( 65 y más, PAL, PROSPERA y PET), los cuales en forma conjunta, alcanzaban una cobertura del 65\%; mientras que los programas de fomento productivo, impulsados por la SAGARPA, apenas atendían el 34.6\% de estos productores. Por otro lado, los apoyos de la CNDPI solo incorporaban al $0.4 \%$ de la población encuestada. Es relevante señalar que el programa PROSPERA contaba con una mayor cobertura de los programas de SEDESOL (50.8\%) en las pequeñas unidades de producción de estos seis estados analizados.

En los resultados del trabajo en 22 comunidades de los seis estados del sur y sureste, se advierte que el PROSPERA no influyó en mejorar los hábitos alimenticios, ni la dieta de los "beneficiarios". Así mismo, se encontró que, en la mayoría de las comunidades, los sujetos de apoyo no siempre eran los más nece- 
sitados (pobres) del lugar. En muchos casos, las personas en situación de mayor pobreza no llegaban a enterarse del programa, ni de los calendarios de asignación y distribución de los beneficios. En otros casos, como el programa de comedores comunitarios o escolares, no se habilitaron en las localidades de los municipios considerados de alta marginación; y en algunos, los comedores se ubicaron en las cabeceras municipales, no en las comunidades rurales con mayores carencias.

A pesar de los distintos apoyos a la agricultura en México, el país es el principal importador mundial de maíz con 16.5 millones de toneladas en 2018, es deficitario en trigo, arroz y frijol, además de ser uno de los principales países importadores de leche en polvo (SADER, 2019). Ante esto, al inicio del sexenio del presidente López Obrador (2018-2024), se emprende la llamada "Cuarta transformación” o 4T, que implica reformas estructurales de las políticas hacia el campo para atender a la población más desprotegida y olvidada. También se propusieron cambios en la estructura y funcionamiento de la SAGARPA, ahora Secretaría de Agricultura y Desarrollo Rural (SADER), y de sus políticas, así como la ejecución de nuevos programas. Así como la SEDSOL, pasó a ser la Secretaría del Bienestar (SB). En ambas dependencias, se cuenta con dos programas, de los 30 estelares de la 4T, el Programa Produciendo para el Bienestar (PpB) en la SADER; y el Programa Sembrando Vida (PSV), en la SB

Los nuevos programas se encuentran en una situación que puede enunciarse en la siguiente pregunta: “Vino nuevo en viejos odres?”, es decir, programas de transformación con los "viejos técnicos para la asistencia y asesoría", "con los anteriores esquemas financieros", "con las viejas tecnologías", "con los deteriorados canales de mercado y coyotaje", con la participación de los viejos "líderes" políticos o "sociales", "con la vieja visión sectorial de las políticas públicas", etc. (Baca et al., 2019).

A continuación, se analiza la información disponible para estos dos programas del nuevo sexenio: Producción para el bienestar y Sembrando vida.

\subsection{Producción para el bienestar (PpB)}

Dentro de los programas estratégicos de la SADER, se encuentra el denominado Producción para el bienestar (SADER, 2019); éste se enfoca en la autosuficiencia alimentaria y la coordinación de programas, pretende ser integral porque se conjuga con otros de la misma instancia como: Crédito a la palabra, Precios de garantía, Programa nacional de fertilizantes, e incluso el de Crédito ganadero. Su objetivo es lograr la autosuficiencia alimentaria en maíz, frijol, arroz, trigo panificable, leche, productos cárnicos y pescado; esto a través de apoyar a pequeños y medianos productores.

Este programa, gestionado por la Subsecretaría de Alimentación y Competitividad, plantea básicamente: a) incorporar predios hasta de 20 has, que cultiven maíz y frijol en productores ya identificados en 2018 y que se ubiquen en localidades marginadas y con población indígena, b) dar subsidios y apoyos por hectárea, c) establecer esquemas de capacitación y acompañamiento técnico, entre otros.

De lo anterior, es posible señalar que varios de los planteamientos sobre agricultura familiar multifuncional y PP, descritos en el estudio realizado por Baca et al. (2019), se encuentran reflejados en esta propuesta de PpB: eliminar varios de los programas repetidos, reducir las reglas de operación, para que sean sencillas y fáciles de entender entre los beneficiarios, que se atienda en forma prioritaria a las familias con mayor índice de pobreza y marginación, incluidos los grupos indígenas; que los programas apunten a la integralidad y a la instrumentación de cadenas productivas, que agreguen valor y atiendan desde la 
producción hasta el consumo, incluyendo la parte financiera y de organización.

No obstante, aún existen más dudas que certezas en este programa, algunas de las preguntas que surgen para visualizar la efectividad y eficacia de aplicación en UAMF son: ¿Quién y de qué forma se ha levantado el censo de productores?, ¿la entrega de los apoyos será verificable?, ¿en tiempo y forma?, ¿los beneficiarios tendrán la capacidad bancaria para ejercerlos?, ¿qué sucedería si los beneficiarios incumplen con sus compromisos? En cuanto a los insumos: ¿ Se tiene la capacidad para la producción y entrega de la semilla requerida?, ¿qué tipo de semilla se distribuirá: certificada o criolla?; de los fertilizantes: ¿Quién los producirá? ¿se continuarán importando?, ¿qué tanto se apoyará la producción y uso de los biofertilizantes?, ¿que hay sobre la política de los pesticidas? Y sobre la asistencia técnica: ¿A quiénes se capacitará? ¿quién lo hará? La contratación ¿Será individual o en grupos, con agencias de desarrollo? Los técnicos actuales ¿Se capacitarán?, ¿serán locales, es decir, con ubicación en los municipios que trabajarán? ¿Cuánto tiempo trabajarán en gabinete y en campo?, ¿contarán con apoyo de técnicos comunitarios?, ¿quién y cómo seleccionarán y capacitarán a esos técnicos comunitarios?

\subsection{Avance de la política pública PpB}

La SADER, publicó en septiembre de 2019, una convocatoria para "Servicios de extensionismo en el territorio de un CADER" de tipo agrícola, pecuario y ciencias sociales, el proceso de selección aún no concluía para el 1 de octubre 2019 y, además, no se conocía cuándo iniciarían los trabajos, ni los lineamientos de operación.

En seguimiento del proyecto mencionado: PESA y CNCH sinergias para potenciarlas, se realizó una segunda recopilación de información de abril a septiembre del 2019, en esta etapa se realizaron un total de 105 entrevistas semiestructuradas a los productores (de tres a cinco de cada comunidad), con los que se trabajó, se investigó sobre la información que tenían de los programas "Produciendo para el bienestar" y "Sembrando vida".

Se indagó sobre el acceso y aplicación (o reglas de operación), si habían sido encuestados y eran parte del padrón correspondiente y, si ya habían recibido las tarjetas y beneficios de los mismos.

El 48\% de los entrevistados manifestó que solo conocía los programas por la publicidad en la radio o la televisión, que no habían recibido aún visitas para empadronarlos y mucho menos su tarjeta o beneficios; y no sabían cómo y dónde acceder a este programa. Otro 25\% informó que había acudido al CADER u oficina del DDR a pedir información y que solo les habían dado folletos informativos y les comentaron que tuvieran paciencia, que los irían a encuestar y les darían instrucciones sobre cómo acceder a éste y otros programas. Menos del 30\% declaró que sí conocían el programa, que ya los habían encuestado e inscrito en el padrón, pero aún no recibían su tarjeta o beneficios.

Información obtenida en campo, indicaba que al mes de septiembre de 2019, la mayoría de los productores entrevistados (productores pequeños con menos de 5 has) en varios municipios de Chiapas, Oaxaca, SLP, Quintana Roo, Veracruz y Yucatán, carecía de información suficiente y precisa respecto al programa, su operación y, lo más importante, los mecanismos para que se les considerara sujetos de apoyo y recibieran los beneficios.

De los avances del programa podemos señalar que cuenta con el apoyo de la Universidad Autónoma Chapingo (UACh) para el diseño y primeras etapas del programa, así como el diseño de programa de 
asistencia técnica en agroecología, la cual está siendo acompañada por el Instituto Nacional de Investigaciones Forestales, Agrícolas y Pecuarias (INIFAP). Y qué este año (2020) ha puesto a funcionar ese esquema de agroecología.

Se contrataron y capacitaron técnicos que trabajan en pareja, uno "productivo" y otro "social", así mismo se rescatan los saberes campesinos y se intercambian estos conocimientos en "escuelas de campo", siguiendo la experiencia al respecto de la UACh

\subsection{Programa Sembrando vida (PSV)}

Sobre este emblemático y estratégico programa de la 4T dirigido en especial a los estados del Sursureste (SB, 2019), los lineamientos de la Secretaria del Bienestar (SB) señalan:

El Programa incentivará a los sujetos agrarios a establecer sistemas productivos agroforestales, el cual combina la producción de los cultivos tradicionales en conjunto con árboles frutícolas y maderables, y el sistema de Milpa Intercalada entre Árboles Frutales (MIAF), con lo que se contribuirá a generar empleos, se incentivará la autosuficiencia alimentaria, se mejorarán los ingresos de las y los pobladores y se recuperará la cobertura forestal de un millón de hectáreas en el país. Asimismo, el Programa impulsará la participación efectiva de mujeres y hombres con la intención de contribuir a cerrar brechas de desigualdad por género y etnia en el acceso a los recursos. (SB, 2020, p. 4)

Los lineamientos: están enfocados a una población objetivo (UAMF con 2.5 has disponibles), cuentan con criterios de priorización en ocho estados del país: Campeche, Chiapas, Durango, Puebla, Quintana Roo, Tabasco, Veracruz y Yucatán. Asimismo, se señala que se dará prioridad a jóvenes en edad productiva, a mujeres campesinas e indígenas, a sujetos agrarios que no participen en otro programa federal con fines similares y a sujetos agrarios con unidades de producción propias.

En el diseño de este programa también se reflejan algunos de los planteamientos ubicados en la segunda parte del estudio realizado en 2017-2019 (Baca et al., 2019), con el enfoque de agricultura familiar multifuncional, como son: la propuesta del Maíz Intercalado con Árboles Frutales (MIAF), la integración, con voz y voto de las mujeres, de la población indígena; el rescate del patrimonio cultural y los conocimientos de los adultos mayores, así como de los sistemas agroecológicos tradicionales. Adicionalmente, coincide con la recomendación de disminuir el número de programas y aumentar la calidad del gasto gubernamental en inversiones sólidas y mejor enfocadas, entre otras.

Para mediados del 2019, también se planteaban diversos cuestionamientos en la puesta en marcha de este programa, como: ¿No se ubica dónde está el diagnóstico para este programa, si es que se realizó?, ¿cuál es el problema central que trata de resolver?, ¿quién y de qué forma se efectúa el censo de productores?, ¿la entrega de los apoyos será verificable en tiempo y forma?, ¿los beneficiarios tendrán la capacidad para ejercerlos?, ¿los productores estarán en condiciones de modificar sus rutinas para atender los trabajos en los viveros y demás?, ¿se tomará en consideración la experiencia de instituciones de investigación y enseñanza con amplia experiencia en el sistema MIAF, como el Instituto Nacional de Investigaciones Forestales, Agrí- 
colas y Pecuarias-INIFAP-, la Universidad Autónoma Chapingo y el Colegio de Posgraduados?

En cuanto los insumos, también es posible plantear diversos cuestionamientos: ¿Existe capacidad para producir y entregar la semilla y plantas requeridas? El tipo de semilla a distribuir ¿será certificada o criolla?, ¿qué tipo de árboles se sembrarán? La elaboración y aplicación de biofertilizantes ¿se apoyará?, ¿qué política hay respecto a los pesticidas? De la asistencia técnica: ¿Habrá capacitación a los técnicos que ya existen, en qué aspectos y quiénes? Los contratos ¿serán individuales, por equipo, ADR, o similar?, ¿los técnicos serán de las localidades?, ¿cuánto tiempo trabajarán en gabinete y cuánto en campo?, ¿tendrán apoyo de técnicos comunitarios?, ¿quién y cómo seleccionarán y capacitarán a esos técnicos comunitarios? ¿el Programa se efectuará en las condiciones previstas? considerando que los productores tienen otro tipo de rutinas establecidas, etc.

\subsection{Avance de la política pública PSV}

De las entidades en estudio que se analizaron durante el periodo 2017 a 2019, el programa de Sembrando vida solo se ha implementado en parte de Chiapas, Quintana Roo, Veracruz y Yucatán; en Oaxaca y SLP se supone iniciaría en 2020.

Un aspecto relevante a señalar es que, en las entidades donde se registró el inicio del Programa, del total de productores entrevistados, un $50 \%$ señaló que debieron desmontar y preparar entre 1 y 1.5 has adicionales a las que venían trabajando de forma anual; ya que para ser sujetos de apoyo se requiere que los predios sumen 2.5 has por productor, lo cual representa una contradicción al trabajar con el MIAF o árboles forestales, ¿ desmontar para sembrar árboles?

Fuera de este aspecto, los productores que ya estaban dentro del programa manifestaron su aceptación y beneplácito, también saben que habrá resultados este año como la cosecha de maíz, y otros serán a mediano plazo como los árboles frutales o maderables, además de los beneficios ambientales de retención de humedad y suelo, regeneración del suelo y fauna local.

Así mismo, este programa cuenta con un consejo consultivo integrado por especialistas de diversas instituciones. Las reglas de operación de aceptaron y publicaron el 31 de marzo de este año (2020).

Para la operación del PSV, en 2019, se contrataron: "4 Coordinadores(as) Regionales, 13 Coordinadores(as) Territoriales, 5 Especialistas Productivos y Sociales, 230 Facilitadores(as) Comunitarios, 1,150 Técnicos(as) Sociales, 1,150 Técnicos(as) Productivos además de los Becarios(as) del programa Jóvenes Construyendo el Futuro" (CONEVAL, 2020, p. 32)

Se contrataron parejas de técnicos que trabajan de forma conjunta uno "productivo" y otro "social". También cuentan con el apoyo de técnicos comunitarios contratados por el Programa de Jóvenes construyendo el futuro de la STPS. Así mismo, para el rescate del conocimiento tradicional se han implementado Centros de Aprendizaje Campesino (CAC).

Para el abasto de los árboles, en el 2019 se utilizaron los viveros forestales militares de la SEDENA, este año (2020), se continúa con el apoyo de estos viveros, pero se han implementado viveros comunitarios.

Los recursos con que cuenta el PSV son: "De acuerdo con el Presupuesto de Egresos de la Federación el presupuesto asignado del PSV en 2019 fue de 15,000 millones de pesos, para 2020 se le asignaron 28,504.9 millones de pesos. Lo que representa un incremento del 90\% en términos nominales" 
(CONEVAL, 2020, p. 16). Lo cual representa el presupuesto más alto para un programa "productivo", aunque se ubique en el sector social de la SB.

Con lo cual, el PSV planeó apoyar, al cierre de 2020, “a campesinos en zonas rurales para que, mediante el establecimiento de proyectos agro-productivos, bajo sistemas agroforestales en localidades con mayor degradación forestal y marginadas socialmente, sean sembradas un millón setenta y cinco mil hectáreas, recuperando la cobertura vegetal en beneficio de 400 mil campesinos en esas zonas". (CONEVAL, 2020, p. 35) (subrayado de los autores).

\section{Discusión}

Como se señala en el título del trabajo: “de la dispersión a la concentración de las PP para el campo", las políticas de la 4T al sector rural, transitan de una gran dispersión de programas para los pequeños agricultores en el campo a una concentración en sólo siete programas agrupados en la Subsecretaría de Alimentación y Competitividad, de la SADER, que pretenden ser coordinados a su vez en el programa Producción para el Bienestar; así como el Programa Sembrando Vida, como esquemas emblemáticos y estratégicos de la 4T. En la práctica, no se abordó un proceso de consulta, discusión y planeación del programa, como lo plantean los teóricos de las PP; nuevamente se recurrió solo a los considerados "expertos" para hacer este trabajo, y quedó fuera la gran mayoría de los futuros "beneficiados" o "sujetos de apoyo". Lo cual se refleja en el desconocimiento que los productores dicen tener sobre este programa, aun cuando ya inició y avanzó el ciclo agrícola, ni que decir de sus beneficios y asistencia técnica al respecto.

Así mismo, el nombramiento de un "super delegado", que rinde cuentas directamente al Presidente, deja fuera de muchos aspectos tanto a los productores, como a funcionarios locales y estatales, concentrando las decisiones nuevamente en el centro.

Si bien los funcionarios de la SADER y de la Secretaría del Bienestar están constantemente en gira por el país, no es suficiente para resolver un sinnúmero de tareas y problemas cotidianos que se presentan en la operación de los programas.

Por ello, es importante reflexionar en que las políticas públicas basadas en evidencias pueden contribuir a una mayor eficiencia de los programas destinados al sector rural del país, ya que este enfoque "preconiza la necesidad de que los programas sociales, tratamientos, intervenciones, servicios y acciones en general que se apliquen a cualquier contexto, deberían estar avalados por la mejor evidencia científica posible" (Buchanan, 2009, p. 45).

El enfoque basado en evidencias se debe de construir, tal como lo señala Bracho (2010):

La evidencia no se identifica con la información existente, sino que es un dato que se construye tanto desde los sistemas de información de la administración pública como desde los propios investigadores en su actividad evaluativa y que adquiere un significado argumentativo en su uso político. la evidencia es tal en el preciso momento en que está disponible, se difunde y es utilizada para retroalimentar a las políticas públicas. (p. 331) 
Las evidencias obtenidas en el trabajo de campo realizado en seis estados con unidades familiares de producción campesina menores a 5 hectáreas nos muestran que la definición de programas de política pública que superen los problemas burocráticos y que desarrollen capacidades e incentiven la producción para un mayor bienestar de las familiares rurales es un tema aún pendiente. Es decir, a nivel de unidad de pequeña unidad de producción sigue persistiendo un bajo conocimiento de la oferta de programas del gobierno, por lo que la cobertura es limitada y su implementación integra más dudas que resultados. De tal forma que como señala Velásquez (2018):

Las políticas y programas necesitan identificar intervenciones efectivas que tengan base científica, establecer metas y estrategias de expansión de coberturas de estas intervenciones, evaluar las restricciones que impiden que las intervenciones y servicios lleguen a los más vulnerables, analizar el costo de incrementar las coberturas de estas intervenciones, el presupuesto disponible y el presupuesto adicional para expandir las coberturas. (p. 371)

\section{Conclusión}

En la actualidad en México, aproximadamente la mitad de la población se encuentra en alguna situación de pobreza, a pesar de los presupuestos de miles de millones de pesos aprobados en los últimos años, y a la existencia de políticas públicas enfocadas para asistencia social y desarrollo productivo del sector rural. Las políticas se concertaron, hasta hace poco en más de 150 programas que, en el discurso, se orientaron a la atención de la población vulnerable en el campo. Sin embargo, en la práctica, y de acuerdo con las categorías de análisis planteadas en el estudio realizado, se operaron menos del 10\% de los mismos. Es decir, llegaban a las familias, en promedio, únicamente dos programas, y regularmente predominaba el de asistencia social, pero tampoco se lograba en la práctica alcanzar los resultados esperados, por ejemplo en el asunto de una mejor alimentación.

En un entorno económico neoliberal, donde se supone que el mercado se encargaría de lograr una distribución equitativa resulta claro, con base en el estudio y los análisis aquí presentados, que las políticas públicas y los programas que hasta hace poco se promovían no debían continuar de la misma manera.

Al parecer tal situación ha sido identificada y se han planteado cambios que, en la propuesta, se orientan de forma más apropiada para resolver las situaciones de la realidad rural del país, sobre todo en la referida a las pequeñas unidades de producción familiar. Estos nuevos esquemas parecen contener algunos puntos fundamentales que se advirtieron en los resultados del trabajo de investigación. Ante esto, es importante continuar el estudio para conocer si en la práctica se tendrán los alcances y resultados esperados, y saber si se logrará la promoción autogestiva de un verdadero desarrollo de las capacidades productivas, técnicas y organizativas de las familias y con ello la superación de la pobreza y el acceso a mejores niveles de bienestar.

Sobre la base de los resultados obtenidos de estos programas en el futuro, verificar si la selección de los productores en los programas es realizada a partir de la existencia y conocimiento de lo que demanda el programa, si alcanza una cobertura que impacte a nivel de territorio y que su implementación en campo detone un cambio de los apoyos gubernamentales en la producción primaria que incida en una mejoría del bienestar de las familias rurales del país. Por lo tanto, hay que esperar el avance y la obtención de 
resultados, ya que de momento no se conoce mucho al respecto y en algunos casos se han abandonado otras iniciativas que empezaban a funcionar.

Algunas recomendaciones emanadas del presente estudio son las siguientes: mejorar la coordinación de políticas públicas y de sus programas, reducir el número y fortalecer los programas que se reestructurarán, sobre todo los dirigidos a pequeños productores, que el diseño y ejecución considere la planeación participativa comunitaria. Con la $4 \mathrm{~T}$ observamos que podría generarse una polarización mayor, no solo por la reestructuración y cambios a nivel de secretarías (SAGARPA por SADER, SEDESOL por la Secretaría del Bienestar -SB-), sino además por la gran reducción de disposiciones hacia el campo, concentradas alrededor de Producción para el Bienestar y el Programa Sembrando vida, así como por la centralización de las decisiones y la ejecución de los recursos.

\section{Referencias}

Aguilar, V. L. F. (1992a). Estudio introductorio. En Aguilar V. L. F. (Ed.), La Hechura de las políticas (pp. 15-84). Porrúa.

Aguilar, V. L. F. (1992b). Estudio introductorio. En Aguilar V. L. F. (Ed.), El Estudio de las políticas públicas (pp. 15-74). Porrúa.

Aguilar, V. L. F. (1993). Estudio introductorio. En Aguilar V. L. F. (Ed.), Problemas públicos y agenda de gobierno (pp. 15-72). Porrúa.

Aguilar, V. L. F. (2008). Marco para el análisis de las políticas públicas. Administración y cidadanía: revista da Escola Galega de Administración Pública, 3(2), 9-28. http://dialnet.unirioja.es/servlet/ oaiart?codigo $=2898247$

Baca, M. J. y Cuevas, R. V. (2018). Desvinculación de las políticas públicas en el campo mexicano. Andamios, Revista de Investigación Social, 15(38), 319-338. http://dx.doi.org/10.29092/uacm.v15i38.662

Baca, M. J., Cuevas-Reyes, V. y Barradas-Miranda, P. (4-7 de junio de 2019). Las encrucijadas de las transformaciones de las políticas al campo: ¿Vino nuevo en viejos odres? [conferencia]. XII Congreso Nacional de la AMER. UNAM, México.

Barradas, M. P. (24-27 de abril de 2018). Un diagnóstico de género y organizaciones rurales [conferencia]. VI Congreso Internacional y XX Congreso Nacional de Ciencias Agronómicas. Chapingo, Estado de México.

Bracho, T. (2010). Políticas basadas en evidencia. La política pública como acción informada y objeto de investigación. En Merino M. y Cejudo G. (Ed.), Problemas, decisiones, soluciones. Enfoques de política pública (pp. 291-321), FCE/CIDE.

Buchanan, A. (2009). Política y práctica social basada en la evidencia: ¿Una nueva ideología o un imperativo de Derechos Humanos? Revista Trabajo Social, 76, 7-16. https://repositorio.uc.cl/bitstream/ handle/11534/6518/000534182.pdf? sequence=1\&isAllowed=y

Consejo Nacional de Evaluación de la Política de Desarrollo Social (CONEVAL). (2016). Evaluación integral del desempeño de los programas federales de ayuda alimentaria y generación de capacidades, 2014- 2015. https://www.coneval.org.mx/Evaluacion/IEPSM/IET/Documents/2014/Ayuda_Alimentaria.pdf

Consejo Nacional de Evaluación de la Política de Desarrollo Social (CONEVAL). (2020). Evaluación de 
diseño con trabajo de campo del Programa Sembrando Vida 2019-2020. https://www.coneval.org. mx/Evaluación/SB-Programa-Sembrando-vida-2019-2020-1.pdf

Flores, C. P. (2013). El enfoque de la política basado en la evidencia. Análisis de su utilidad para la educación de México. Revista Mexicana de Investigación Educativa, 18(56), 265-290. http://www. redalyc.org/articulo.oa?id=14025581012

González, M. M. (2005). El estudio de las políticas públicas: un acercamiento a la disciplina. Quid Iuris, 1(2), 99-118.

Gutiérrez, L. E., Piazza, M., Gutiérrez-Aguado, A., Hijar, G., Carmona, G., Caballero, P., Reyes, N., Canelo, C., Aparco, J. P., Tejeda, A. R., Bolaños-Díaz, R., Saravia, S. y Gozzer, S. (2016). Uso de la evidencia en políticas y programas de salud aportes del instituto nacional de salud. Revista Peruana de Medicina Experimental y Salud Pública, 33(3), 580-584. https://doi.org/10.17843/ rpmesp.2016.333.2308

Lasswell, H. (1992). La orientación hacia las políticas. En Aguilar V. L. F. (Ed.), El estudio de las políticas públicas (pp. 79-103). Porrúa.

Davies, D. (19 de febrero de 2004). Is Evidence-Based Government Possible? [conference], by Jerry Lee. 4th Annual Campbell Collaboration Colloquium, Washington, D.C. http://citeseerx.ist.psu.edu/ viewdoc/download?doi=10.1.1.545.364\&rep=rep1\&type=pdf

Meny, I. y Thoenig, J. C. (1992). Las Políticas Públicas, Versión española a cargo de Francisco Morata. Ariel. (Original publicado en 1989)

Oszlak, O. y O’Donnell, G. (1995). Estado y políticas estatales en América Latina: hacia una estrategia de investigación. Redes, 2(4), 99-128. https://www.redalyc.org/articulo.oa?id=907/90711285004

Peters, G. (2000). Agenda Setting and Public Policy. En Tadao M (Ed.), The Science of Public Policy, Essential Readings in Policy Sciences II, Volume V. (pp. 469-493). Routledge.

Secretaría del Bienestar (SB). (2019). Programa Sembrando Vida. Diario oficial.

https://www.gob.mx/bienestar/acciones-y-programas/programa-sembrando-vida

Secretaria del Bienestar (SB). (2020). Acuerdo por el que se emiten los lineamientos de operación del Programa Sembrando Vida. Diario oficial. http://dof.gob.mx/nota_detalle.php?codigo=5590695 \&fecha $=30 / 03 / 2020$

Secretaria de Agricultura y Desarrollo Rural (SADER). (2019). Programa Produciendo para el Bienestar. Diario oficial https://www.gob.mx/cms/uploads/attachment/file/437378/LINEAMIENTOS_ DOF_23_ENERO_2019_DOF.pdf

Sutcliffe, S. y Court, J. (2006). Herramientas para Diseñadores de Políticas Públicas en Países en Desarrollo. https://www.odi.org/sites/odi.org.uk/files/odi-assets/publications-opinion-files/3520.pdf

Valles, J. M. (2000). Ciencia política: una introducción. Ariel.

Velásquez, A. (2018). Investigación en políticas y sistemas de salud para la gestión basada en evidencias. Revista Peruana de Medicina Experimental y Salud Publica, 35(3), 371-372. https://dx.doi. org/10.17843/rpmesp.2018.353.3978

Villalobos, A. V. M. (20 de julio de 2018). Seguridad, soberanía y autosuficiencia alimentaria. La jornada del campo, núm. 129. https://www.jornada.com.mx/2018/06/16/cam-seguridad.html

Wastell, D. G. (2006). Information systems and evidence-based policy in multi-agency networks: The micro-politics of situated innovation. The Journal of Strategic Information Systems, 15(3), 197-217. 

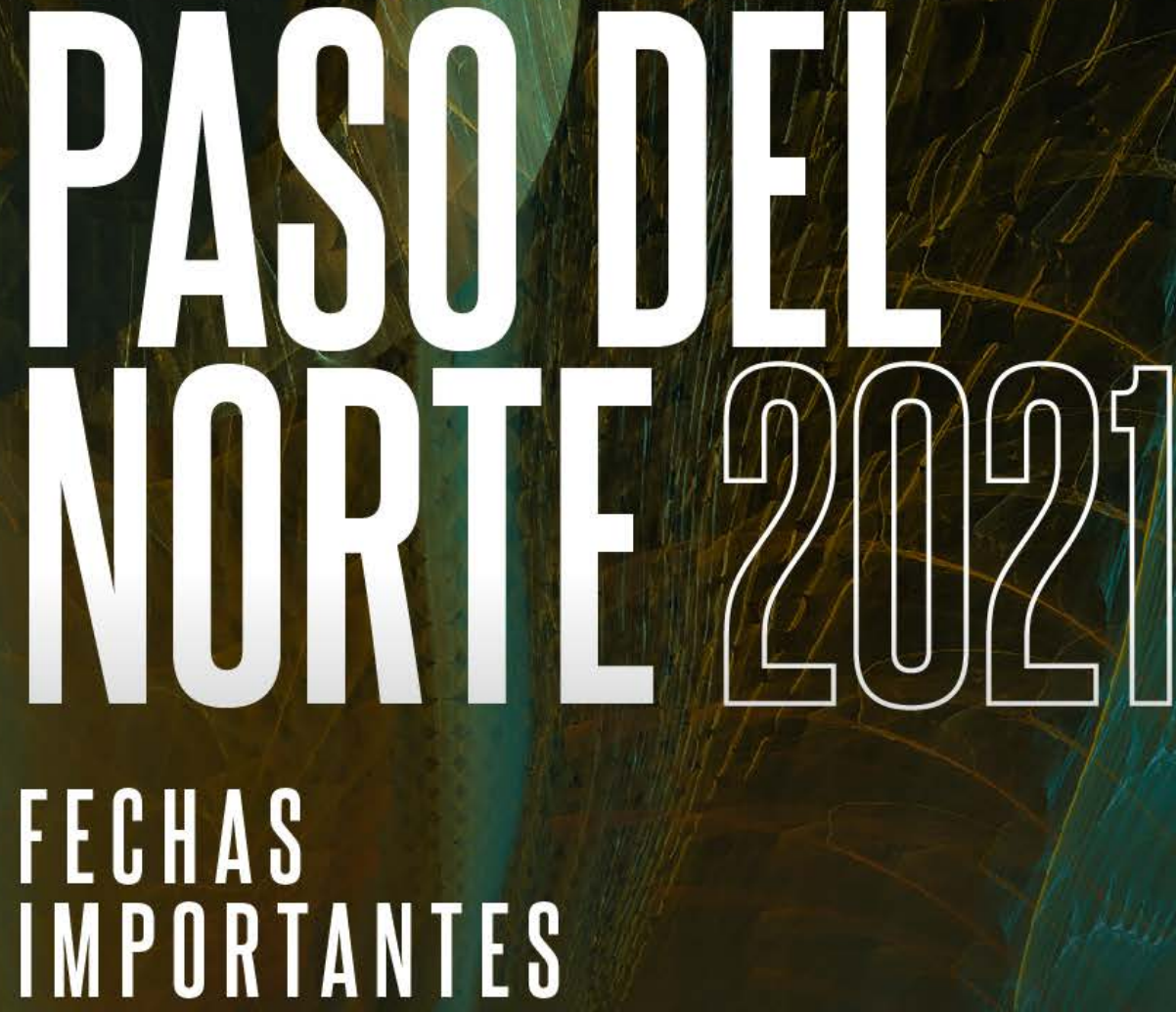

Recepción de trabajo

Del 15 de febrero al 7 de junio

Publicación de propuestas aceptadas 30 de julio

\begin{tabular}{ll}
\hline Fecha límite envío ponencias in extenso & $\mathbf{3 0}$ de septiembre \\
\hline Fecha límite de inscripción para ponentes & $\mathbf{8}$ de octubre \\
\hline Publicación del programa del Congreso & $\mathbf{2 2}$ de octubre \\
\hline Realización del Congreso & del $\mathbf{8}$ al $\mathbf{1 1}$ de noviembre \\
\hline Fecha límite para trámite de constancias & $\mathbf{3 0}$ de noviembre
\end{tabular}

Fecha límite para trámite de constancias

30 de noviembre

Registro de trabajos:

http://info.uacj.mx/congresocs2021 